\title{
Object-Oriented Library of Switching Moving Boundary Models for Two-phase Flow Evaporators and Condensers
}

\author{
Javier Bonilla $^{a} \quad$ Luis J. Yebra $^{a} \quad$ Sebastián Dormido $^{b} \quad$ François E. Cellier $^{c}$ \\ ${ }^{a}$ Centro de Investigaciones Energéticas MedioAmbientales y Tecnológicas (CIEMAT) \\ Plataforma Solar de Almería (PSA), Almería, Spain \\ ${ }^{b}$ National Distance Education University (UNED), \\ Department of Computer Science and Automatic Control, Madrid, Spain \\ ${ }^{c}$ Swiss Federal Institute of Technology (ETH Zurich), \\ Department of Computer Science, Zurich, Switzerland
}

\begin{abstract}
This paper discusses a Modelica library of switching moving boundary models for two-phase flow heat exchangers: evaporators and condensers. The equationbased object-oriented modeling paradigm has been considered by means of designing basic models applying the conservation laws for each flow state: subcooled liquid, two-phase flow and superheated vapor. Evaporator and condenser models have been developed by interconnecting the basic models and including mechanisms to switch between different configurations: general, flooded and dry evaporators and condensers. Finally, simulation results are presented by an integrity and stability test case.
\end{abstract}

Keywords: Moving boundary model; switching; two-phase flow; evaporator; condenser

\section{Introduction}

Heat exchangers play a very important role in industry; the modeling and control of these elements is a key part in the process plant control. Two of the most common discretization approaches used in fluid dynamic modeling are the finite-volume distributedparameter method [21] and the moving-boundary lumped-parameter method [8]. Dynamic modeling is always a challenging task in which the trade-off between accuracy and speed must be evaluated depending on the purpose of the model. Moving boundary models are low-order and much faster models than finite volume models; additionally they can describe the dynamic behavior of evaporators and condensers with high accuracy [1]. In the context of real-time simulation, dynamic system optimization and model-based control, where fast computation is required, the moving boundary method seems to be appropriate.

The moving boundary method divides the evaporator/condenser in different regions, also called Control Volumes (CVs), depending on the fluid phase. In each $\mathrm{CV}$, the lumped thermodynamic properties are averaged; the barrier is not fixed and it may move between adjacent CVs. The main idea is to dynamically track the lengths of the different regions [16].

The three basic flow states are: subcooled liquid (SC), two-phase flow (TP) consisting of vapor and liquid present simultaneously in the same volume, and superheated vapor ( $\mathrm{SH})$ as represented in Fig. 1. Considering these three basic flow states, compound configurations can be created. Fig. 2 shows these configurations: general, flooded and dry evaporators/condensers.

A state-of-the-art study in moving boundary models for two-phase flow heat exchangers was previously presented in [2] together with a new switching flooded evaporator model. This paper extends previous work by new switching moving boundary models for general/dry evaporators and general/flooded/dry condensers. To the knowledge of the authors, there are three papers related to moving boundary models developed using Modelica [17, 27, 13]. The novelty of this paper is that a strictly object-oriented design is followed.

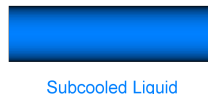

(a)

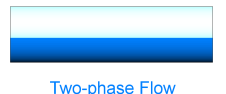

(b)

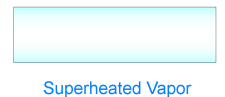

(c)
Figure 1: Basic flow states 


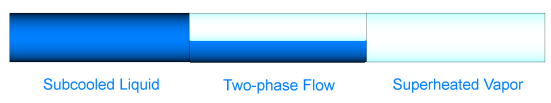

(a) General evaporator

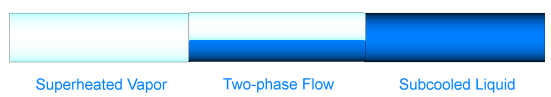

(d) General condenser

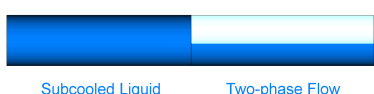

(b) Flooded evaporator

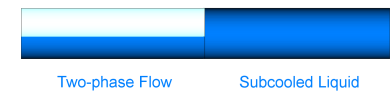

(e) Flooded condenser

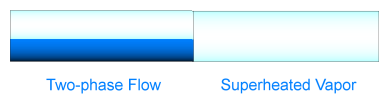

(c) Dry evaporator

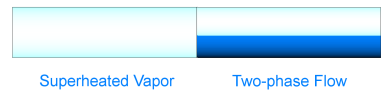

(f) Dry condenser

Figure 2: Evaporator and condenser configurations

\section{Mathematical modeling}

This section first describes the assumptions made in the development of the mathematical models, after that the governing equations in their general form are listed, the one-dimensional CV concept is then introduced, and finally the basic and compound models are explained together with some additional equations required to complete the models.

\subsection{Assumptions}

With the aim of developing a low-order model that reflects the principal dynamics, a number of assumptions have been made: horizontal orientation; onedimensional case; constant pipe cross-sectional area; time-dependent uniform pressure along the evaporator; homogeneous two-phase flow; average properties and time-dependent uniform heat flux per unit length in each $\mathrm{CV}$; negligible gravitational forces; negligible changes in the kinetic energy; negligible viscous stress; heat conduction and radiation in the fluid and heat conduction in the pipe wall are also neglected.

\subsection{Governing equations}

The straightforward way to derive the model equations is from the time-dependent equations for conservation laws. Considering the assumptions presented in the previous section, the differential formulation for the conservation of mass and energy in the fluid are represented by Eqs. 1 and 2, respectively [18]. Eq. 3 [18] defines the conservation of energy in the pipe wall and Table 1 summarizes the nomenclature.

$$
\begin{gathered}
\frac{\partial A \rho}{\partial t}+\frac{\partial \dot{m}}{\partial z}=0, \\
\frac{\partial A \rho u}{\partial t}+\frac{\partial \dot{m} h}{\partial z}=\dot{q}_{i}, \\
A_{w} \rho_{w} c_{p, w} \frac{\partial T_{w}}{\partial t}=\dot{q}_{o}-\dot{q}_{i} .
\end{gathered}
$$

\begin{tabular}{|c|c|c|c|}
\hline Var. & \multicolumn{2}{|l|}{ Description } & Units \\
\hline$t$ & \multicolumn{2}{|c|}{ Time } & {$[\mathrm{s}]$} \\
\hline$z$ & \multicolumn{2}{|c|}{ Spatial coordinate } & {$[\mathrm{m}]$} \\
\hline$A$ & \multicolumn{2}{|c|}{ Cross-sectional area } & {$\left[\mathrm{m}^{2}\right]$} \\
\hline$c_{p}$ & \multicolumn{2}{|c|}{ Isobaric specific heat capacity } & {$[\mathrm{J} /(\mathrm{K} \cdot \mathrm{kg})]$} \\
\hline$\dot{m}$ & \multicolumn{2}{|c|}{ Mass flow rate } & {$[\mathrm{kg} / \mathrm{s}]$} \\
\hline$x$ & \multicolumn{2}{|c|}{ Vapor quality } & {$[-]$} \\
\hline$p$. & \multicolumn{2}{|l|}{ Pressure } & {$[\mathrm{Pa}]$} \\
\hline$\dot{Q}$ & \multicolumn{2}{|c|}{ Heat flow rate } & {$[\mathrm{W}]$} \\
\hline$\dot{q}$ & \multicolumn{2}{|c|}{ Heat flux } & {$\left[\mathrm{W} / \mathrm{m}^{2}\right]$} \\
\hline$\gamma$ & \multicolumn{2}{|c|}{ Void fraction } & {$[-]$} \\
\hline $\bar{\gamma}$ & \multicolumn{2}{|c|}{ Mean void fraction } & {$[-]$} \\
\hline$h$ & \multicolumn{2}{|c|}{ Specific enthalpy } & {$[\mathrm{J} / \mathrm{kg}]$} \\
\hline $\bar{h}$ & \multicolumn{2}{|c|}{ Mean specific enthalpy } & {$[\mathrm{J} / \mathrm{kg}]$} \\
\hline$h^{\prime}$ & \multicolumn{2}{|c|}{$h$ of saturated liquid } & {$[\mathrm{J} / \mathrm{kg}]$} \\
\hline$h^{\prime \prime}$ & \multicolumn{2}{|c|}{$h$ of saturated vapor } & {$[\mathrm{J} / \mathrm{kg}]$} \\
\hline$\rho$ & \multicolumn{2}{|c|}{ Density } & {$\left[\mathrm{kg} / \mathrm{m}^{3}\right]$} \\
\hline $\bar{\rho}$ & \multicolumn{2}{|l|}{ Mean density } & {$\left[\mathrm{kg} / \mathrm{m}^{3}\right]$} \\
\hline$\rho^{\prime}$ & \multicolumn{2}{|c|}{ Density of saturated liquid } & {$\left[\mathrm{kg} / \mathrm{m}^{3}\right]$} \\
\hline$\rho^{\prime \prime}$ & \multicolumn{2}{|c|}{ Density of saturated vapor } & {$\left[\mathrm{kg} / \mathrm{m}^{3}\right]$} \\
\hline$T$ & \multicolumn{2}{|c|}{ Temperature } & {$[\mathrm{K}]$} \\
\hline $\bar{T}$ & \multicolumn{2}{|c|}{ Mean temperature } & {$[\mathrm{K}]$} \\
\hline$\varepsilon$ & \multicolumn{2}{|c|}{ Pipe roughness } & {$[\mathrm{m}]$} \\
\hline Subs. & Description & Subs. & Description \\
\hline$a$ & Inlet to CV & $b$ & Outlet to CV \\
\hline$s c$ & Subcooled & $t p$ & Two-phase \\
\hline$s h$ & Superheated & $w$ & Pipe wall \\
\hline$i$ & Inner to $\mathrm{CV}$ & $o$ & Outer to $\mathrm{CV}$ \\
\hline
\end{tabular}

Table 1: Nomenclature

\subsection{One-dimensional Control Volume}

The moving boundary method is based on the division of the heat exchanger in different CVs. Fig. 3 represents a $\mathrm{CV}$; the lumped thermodynamic properties in the $\mathrm{CV}$ are averaged and they are uniform but time-dependent $(\bar{h}, \bar{T}, \bar{\rho})$; the pressure $(p)$ is not denoted by a mean value, because there is only one time-dependent pressure value for the entire evaporator. The cross-sectional areas $\left(A, A_{w}\right)$ are constant. Each CV has three interfaces or boundaries. One is adjacent to the pipe wall where the thermodynamic properties are also considered in its mean values $\left(\bar{T}_{w}, \bar{\rho}_{w}\right)$. 


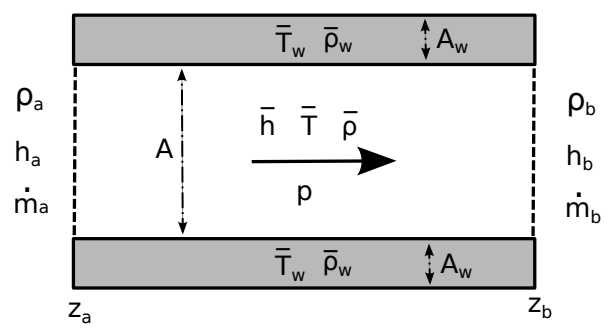

Figure 3: Control Volume (CV)

The other two interfaces connect to adjacent CVs or the inlet or outlet boundaries of the heat exchanger. In Fig. 3 the flow direction is defined by the arrow, so the inlet flow thermodynamic properties correspond to the $a$ subscript variables $\left(\rho_{a}, h_{a}, \dot{m}_{a}\right)$, whereas the outlet flow thermodynamic properties are defined by the $b$ subscript variables $\left(\rho_{b}, h_{b}, \dot{m}_{b}\right)$.

\subsection{Basic Volume Models}

The derivation of the mass and energy balance equations for the $\mathrm{CV}$ models is not presented due to space limitation. From the-state-of-the-art study in two-phase flow moving boundary models useful information was obtained [2]. The derivation of the model is analogous to the developed in $[16,17]$ but not neglecting the mean void fraction time derivative $(d \bar{\gamma} / d t)$, where a new calculation method has been introduced. Additionally, the thermodynamic properties at the boundaries are not fixed to any particular value, by means of considering the density or specific enthalpy of saturated liquid/vapor, so the basic volume models can be used in any evaporator/condenser.

\subsubsection{One-phase Flow Volume Model}

The mass and energy balance equations for the subcooled liquid and superheated vapor $\mathrm{CV}$ models are described by Eqs. 4 and 5 where the subscript $c v$ can be substituted by $s c$ or $s h$ to consider the particular CV.

$$
\begin{aligned}
& A\left(z_{c v} \frac{d \bar{\rho}_{c v}}{d t}+\bar{\rho}_{c v} \frac{d z_{c v}}{d t}\right)+\rho_{a} A \frac{d z_{a}}{d t}-\rho_{b} A \frac{d z_{b}}{d t} \\
& =\dot{m}_{a}-\dot{m}_{b} .
\end{aligned}
$$

$$
\begin{aligned}
& A\left(\bar{\rho}_{c v} \bar{h}_{c v} \frac{d z_{c v}}{d t}+\bar{\rho}_{c v} \frac{d \bar{h}_{c v}}{d t} z_{c v}+\frac{d \bar{\rho}_{c v}}{d t} \bar{h}_{c v} z_{c v}\right)-A z_{c v} \frac{d p}{d t} \\
& +A \rho_{a} h_{a} \frac{d z_{a}}{d t}-A \rho_{b} h_{b} \frac{d z_{b}}{d t}=\dot{m}_{a} h_{a}-\dot{m}_{b} h_{b}+\dot{q}_{i, c v} z_{c v} .
\end{aligned}
$$

\subsubsection{Two-phase Flow Volume Model}

The mass and energy balance equations for the twophase flow CV model are described by Eqs. 6 and 7 . The way the mean void fraction and its time derivative are calculated is described in [2].

$$
\begin{aligned}
& A\left(\frac{d z_{t p}}{d t}\left(\bar{\gamma} \rho^{\prime \prime}+(1-\bar{\gamma}) \rho^{\prime}\right)+z_{t p}\left(\frac{d \bar{\gamma}}{d t}\left(\rho^{\prime \prime}-\rho^{\prime}\right)+\right.\right. \\
& \left.\left.\bar{\gamma} \frac{d \rho^{\prime \prime}}{d p} \frac{d p}{d t}+(1-\bar{\gamma}) \frac{d \rho^{\prime}}{d p} \frac{d p}{d t}\right)\right)+\rho_{a} A \frac{d z_{a}}{d t}-\rho_{b} A \frac{d z_{b}}{d t} \\
& =\dot{m}_{a}-\dot{m}_{b} . \\
& A\left(\frac{d z_{t p}}{d t}\left(\bar{\gamma} \rho^{\prime \prime} h^{\prime \prime}+(1-\bar{\gamma}) \rho^{\prime} h^{\prime}\right)+z_{t p}\left(\frac { d \overline { \gamma } } { d t } \left(\rho^{\prime \prime} h^{\prime \prime}-\right.\right.\right. \\
& \left.\rho^{\prime} h^{\prime}\right)+\bar{\gamma} \frac{d \rho^{\prime \prime}}{d p} \frac{d p}{d t} h^{\prime \prime}+\bar{\gamma} \rho^{\prime \prime} \frac{d h^{\prime \prime}}{d p} \frac{d p}{d t}+(1-\bar{\gamma}) \frac{d \rho^{\prime}}{d p} \frac{d p}{d t} h^{\prime} \\
& \left.\left.+(1-\bar{\gamma}) \rho^{\prime} \frac{d h^{\prime}}{d p} \frac{d p}{d t}\right)\right)-A z_{t p} \frac{d p}{d t}+A \rho_{a} h_{a} \frac{d z_{a}}{d t}- \\
& A \rho_{b} h_{b} \frac{d z_{b}}{d t}=\dot{m}_{a} h_{a}-\dot{m}_{b} h_{b}+\dot{q}_{i, t p} z_{t p} .
\end{aligned}
$$

\subsection{Heat Exchanger Models}

When modeling the compound models (not only one $\mathrm{CV}$ model), additional equations are required besides the $\mathrm{CV}$ governing equations; these equations depend on the kind of heat exchanger and relate the outlet $\mathrm{CV}$ specific enthalpies with the values at saturation conditions.

\subsubsection{Evaporator}

If a general or flooded evaporator is considered (cf. Figs. 2(a) and 2(b)), Eq. 9(a) is required for the subcooled liquid $\mathrm{CV}$, and also the initial value for $h_{b}$ must be set to $h^{\prime}$.

An easy way to accomplish this is to only introduce Eq. 8. However, there is a problem with that approach if switching moving boundaries models are considered.

$$
h_{b}=h^{\prime} .
$$

Suppose that a flooded evaporator is being modeled, where the outlet fluid is two-phase flow; $h_{b}$ for the subcooled liquid CV is not a state variable because it depends on pressure, and therefore Eq. 8 is valid 
and $h_{b}$ is an algebraic variable. However, if the outlet fluid turns into subcooled liquid due to a change in the model inputs, Eq. 8 is no longer valid and $h_{b}$ is a state variable. Such a model is called a variablestructure model. In a variable-structure model the number/type of equations or variables can change, on the other hand a static-structure model implies that the number of equations as well as the number of algebraic and state variables remains the same. Variablestructure models are not currently supported by most modeling and simulations tools (including Modelica tools). Whereas there exist some modeling languages and tools that support variable-structure models, none of the existing variable-structure modeling tools supports the handling of higher-index systems [28]. For that reason, Modelica is still our preferred modeling language, but it must be taken into account that only static-structure models can be simulated.

For this reason, the number of equations must remain the same in all different configurations of our model, and $h_{b}$ for the different CVs must always be a state variable so its value cannot be fixed to any algebraic variable and neither can $h_{a}$, because it is connected to $h_{b}$ from the CV to the left, except for the case of the first $\mathrm{CV}$ where the $h_{a}$ value can be freely establish.

If a general or dry-expansion evaporator is considered (cf. Figs. 2(a) and 2(c)), Eq. 9(b) is required for the two-phase flow CV, and also the initial value for $h_{b}$ must be set to $h^{\prime \prime}$.

\subsubsection{Condenser}

If a general or flooded condenser is considered (cf. Figs. 2(d) and 2(e)), Eq. 9(a) is required for the twophase flow $\mathrm{CV}$, and also the initial value for $h_{b}$ must be set to $h^{\prime}$. If a general or dry condenser is considered (cf. Figs. 2(d) and 2(f)), Eq. 9(b) is required for the superheated vapor $\mathrm{CV}$, and also the initial value for $h_{b}$ must be set to $h^{\prime \prime}$.

$$
\frac{d h_{b}}{d t}=\frac{d h^{\prime}}{d t} \quad(a), \quad \frac{d h_{b}}{d t}=\frac{d h^{\prime \prime}}{d t} \quad(b) .
$$

\subsection{Pipe Wall Model}

The energy balance equation for each pipe wall $\mathrm{CV}$ is described by Eq. 10. This equation is derived in [27], where $T_{w, a}$ and $T_{w, b}$ are the wall temperature values at the interfaces. This approach is closer to the practical situation as it remains continuous and smooth during the switching between different configurations.

$$
\begin{aligned}
& A_{w} \rho_{w} c_{p, w}\left(\frac{d \bar{T}_{w}}{d t}+\frac{\bar{T}_{w}-T_{w, b}}{z_{a b}} \frac{d z_{b}}{d t}+\frac{T_{w, a}-\bar{T}_{w}}{z_{a b}} \frac{d z_{a}}{d t}\right) \\
& =\dot{q}_{o}-\dot{q}_{i} .
\end{aligned}
$$

\subsection{Additional Equations}

Some additional equations are required in order to complete the heat exchanger model. These equations are not detailed here due to space limitations but they can be easily found in the literature [16]. The remaining equations are: the heat flow rates between the pipe wall and the ambient and between the pipe wall and the fluid and the geometric constraints, i.e., the total heat exchanger length and the pipe geometry. The pipe geometry considered in this manuscript has been the cylindrical geometry.

\subsection{Switching}

Switching from one configuration to another implies the disappearance of an existing CV or the appearance of a new one, e.g. when switching from a general evaporator to a flooded evaporator or vice versa. This section elaborates how such transitions are captured by the model. Additional equations for the new $\mathrm{CV}$ may be required. When the $\mathrm{CV}$ is active, its governing equations correspond to the equations described in Sections 2.4.1 or 2.4.2 depending on the fluid phase; however a different set of equations is required to described the CV in its inactive state. This is also explained in this section. It is assumed that the appearance or disappearance of a CV can only occur at the end of the heat exchanger.

\subsubsection{Disappearance of a Control Volume}

A CV disappears (becomes inactive) when Eq. 11(a) becomes true, where $z_{\min }$ denotes a threshold that specifies the minimum length of an active CV. This value cannot be zero in order to avoid structural singularities, therefore the $\mathrm{CV}$ length must be greater that zero. The default value for this parameter has been set to $10^{-6} \mathrm{~m}$.

\subsubsection{Control Volume in an Inactive State}

When any of the CVs is inactive, the mass and energy balance equations (Eqs. 4 and 5 or Eqs. 6 and 7 depending on the $\mathrm{CV}$ fluid phase) are substituted by 
Eqs. 11(b) and 11(c), respectively. These equations guarantee that the $\mathrm{CV}$ is inactive and does not act on the fluid.

$$
\begin{aligned}
& z_{c v}<z_{\min } \quad(a), \quad \dot{m}_{a}=\dot{m}_{b} \quad(b), \\
& \frac{d h_{a}}{d t}=\frac{d h_{b}}{d t} \quad(c), \quad \frac{d z_{c v}}{d t}=0 \quad(d) .
\end{aligned}
$$

Moreover, Eq. 9(a) or 9(b) must be substituted by Eq. 11(d) depending on the inactive CV and on the kind of heat exchanger considered.

\subsubsection{Appearance of a Control Volume}

The event triggering the appearance of a CV depends on the particular CV and also on the kind of heat exchanger.

Evaporator. The superheated vapor CV appears (cf. Figs. 2(a) and 2(c)) when the vapor quality in the twophase flow CV becomes greater than 1.0, Eq. 12(a). The two-phase flow CV appears (cf. Figs. 2(a) and 2(b)) when the outlet specific enthalpy in the subcooled liquid CV becomes greater than the specific enthalpy of saturated liquid, Eq. 12(b).

Condenser. The subcooled liquid CV appears (cf. Figs. 2(d) and 2(e)) when the outlet specific enthalpy in the two-phase flow $\mathrm{CV}$ is lower than the specific enthalpy of saturated liquid, Eq. 12(c). The two-phase flow CV appears (cf. Figs. 2(d) and 2(f)) when the outlet specific enthalpy in the superheated vapor CV becomes lower than the specific enthalpy of saturated vapor, Eq. 12(d).

$$
\begin{array}{ccc}
x>1 & (a), \quad h_{b}>h^{\prime} \quad(b), \\
h_{b}<h^{\prime} & (c), \quad h_{b}<h^{\prime \prime} \quad(d) .
\end{array}
$$

\section{Description of the Library}

This section describes the Modelica library that implements the mathematical models previously described, the MBMs (Moving Boundary Models) library.

\subsection{Library Structure and Interfaces}

Fig. 4(a) shows the main packages that make up the MBMs library, and Fig. 4(b) shows the Components.Water.MBM package in expanded view, where the basic and compound models can be seen. The

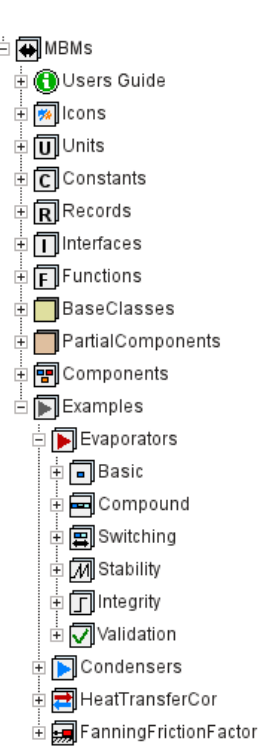

(a) Packages

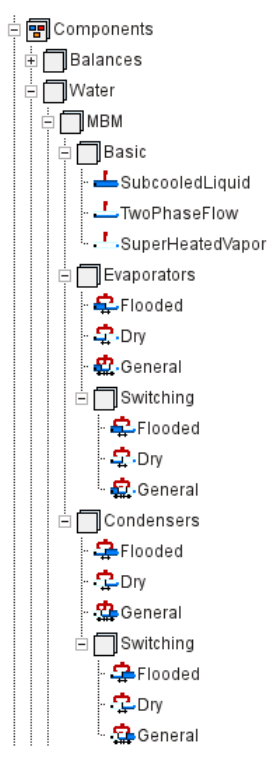

(b) Components
Figure 4: The MBMs library

former do not support switching, whereas the latter do. Modelica Fluid and Modelica Thermal ports have been used throughout in order to define the interfaces in the MBMs library. This guarantees that the MBMs library is compatible with any component from the Modelica standard library 3.2 [20] or from third-party components that also make use of these interfaces.

\subsection{Partial Base Classes}

The most remarkable partial base classes in the MBMs library are: the Volume class, the MultipleVolume class, the HeatTransferCorrelation class and the FrictionFactor class.

\subsubsection{Volume Class}

The Volume class defines the fluid and heat ports, the medium, some additional thermodynamic properties, as well as the state and geometry of the CV. This class is the base class for the basic volume models. The volume class also includes a heat transfer correlation (HTC) and a friction factor model (FFM).

\subsubsection{MultipleVolume Class}

The MultipleVolume class defines two or three CVs that can be redeclared in classes that inherit from it. The CVs are connected through the fluid connectors, and this is the base class for all heat exchangers. We 


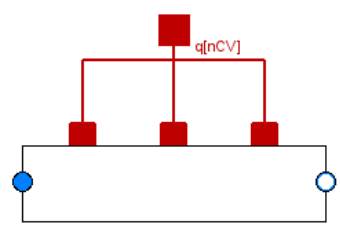

(a) Icon

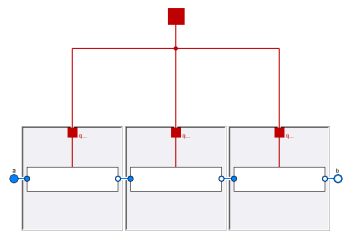

(b) Component diagram
Figure 5: MultipleVolume base class (3 CVs)

followed strictly an object-oriented design for heat exchangers. Figs. 5(a) and 5(b) show the icon and component diagram of the $3 \mathrm{CVs}$ MultipleVolume class.

\subsubsection{Heat Transfer Correlations and Friction Factor Models}

There are two base classes for heat transfer correlations (HTCs) and friction factor models (FFMs). The user can inherit from them to define new HTCs or FFMs. FFMs have been implemented because the Petukhov and Gnielinski HTCs require a friction factor which can be caculated from those FFMs, furthermore there are plans for extending the library with pressure loss. A HTC can be restricted to only one particular fluid phase (one-phase or two-phase) or to only one particular process (evaporation or condensation), if required. Moreover, there are some HTCs for evaporation and FFMs for smooth and rough pipes, always considering turbulent flow, already implemented in the library. They are summarized in Table 2. The implemented HTCs and FFMs have been also adapted to switching in order to avoid discontinuities and numerical problems during the simulation. The HTCs and FFMs have been validated against an independent implementation [26]. The HTC and FFM can be selected in each CV through the GUI. A test case for the implemented two-phase flow HTCs is shown in Fig. 6.

\subsection{Volume Components}

Fig. 7 shows the icons of the subcooled liquid, twophase flow and superheated vapor models. These models inherit from the Volume class and add their particular equations, although the subcooled liquid and the superheated vapor models inherit from an intermediate class in the hierarchy, the OnePhaseVolume class, because both models share the same equations.

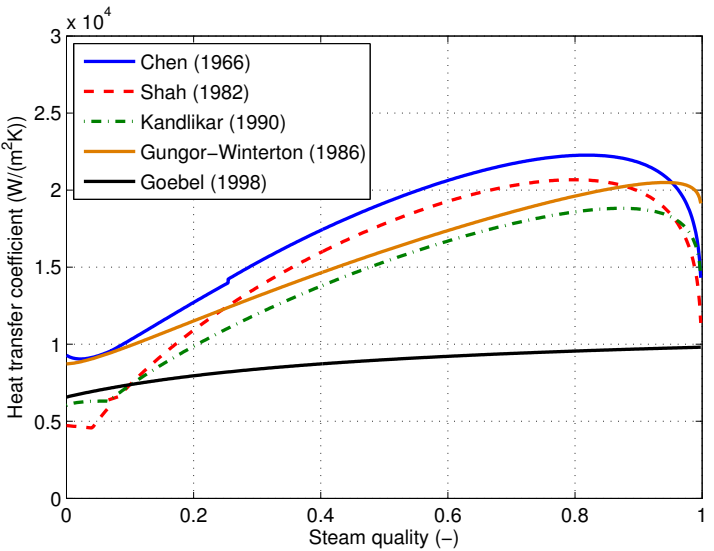

Figure 6: Comparison of two-phase flow HTCs ( $p=3$ $\mathrm{MPa}, \dot{Q}=5,827 \mathrm{Kw}, \dot{m}=0.6 \mathrm{~kg} / \mathrm{s}, \varepsilon=3 \cdot 10^{-5} \mathrm{~m}$ )

\begin{tabular}{|l|l|}
\hline Heat Transfer Correlations & Fluid phase \\
\hline Ideal & any \\
Constant & any \\
[9] Dittus-Boelter (1930) & One-phase \\
[3] Chen (1966) & Two-phase \\
[22] Petukhov (1970) & One-phase \\
[11] Gnielinski (1976) & One-phase \\
[25] Shah (1982) & Two-phase \\
[14] Gungor-Winterton (1986) & Two-phase \\
[19] Kandlikar (1990) & Two-phase \\
[12] Goebel (1998) & Two-phase \\
\hline Fanning Friction Factor Model & Kind of pipe \\
\hline None & - \\
Constant & any \\
[5] Colebrook (1939) & any \\
[4] Chen (1979) & any \\
Explicit simplified Chen (1979) & any \\
[24] Karman-Prandtl (1930) & Rough \\
[7] Denn (1980) & Smooth \\
[15] Haaland (1983) & any \\
\hline
\end{tabular}

Table 2: HTCs for evaporation and fanning FFMs implemented in the MBMs library

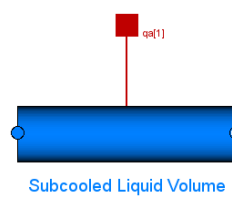

(a)

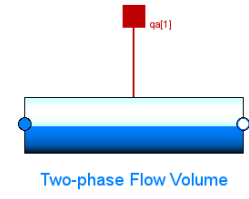

(b)

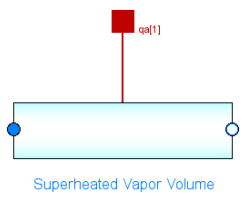

(c)
Figure 7: Volume components 


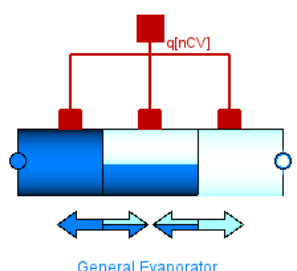

(a)

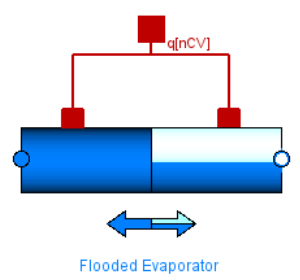

(c)

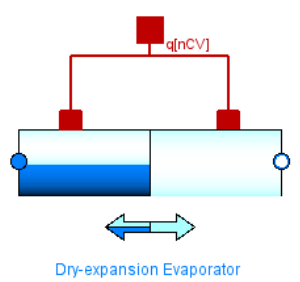

(e)

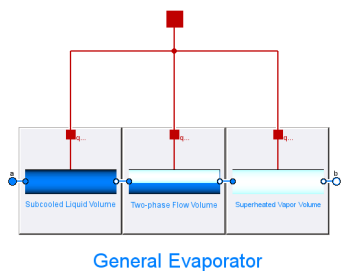

(b)

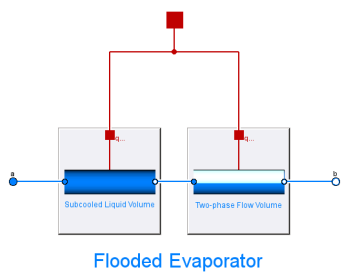

(d)

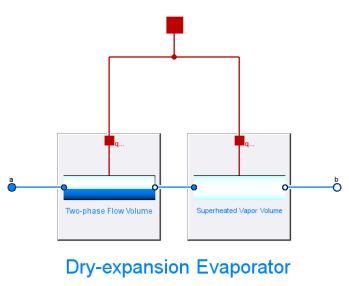

(f)
Figure 8: Evaporator components

\subsection{Heat Exchanger Components}

Redeclaring the partial Volume classes in the MultipleVolume ( 2 or $3 \mathrm{CVs}$ ) class with the volume components: subcooled liquid, two-phase flow and superheated vapor models, evaporators and condenser can be defined.

\subsubsection{Evaporator Components}

Fig. 8 shows the general, flooded and dry-expansion evaporator models. The figures on the left represent the icons whereas the figures on the right are the component diagrams, where the partial Volume classes have been redeclared appropriately.

\subsubsection{Condenser Components}

For condensers, the situation is the same, but changing the order of the interconnected basic volumes models. Fig. 9 shows the general, flooded and dry condenser icons and component diagrams.

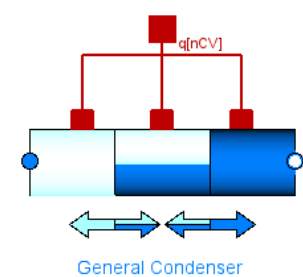

(a)

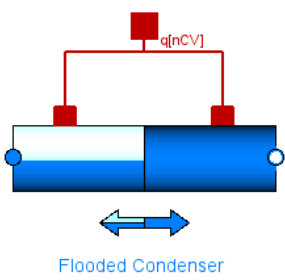

(c)

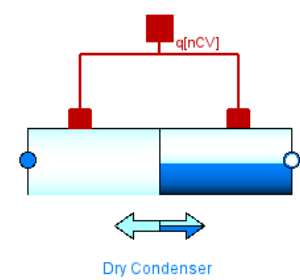

(e)

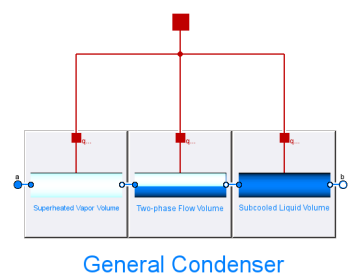

(b)

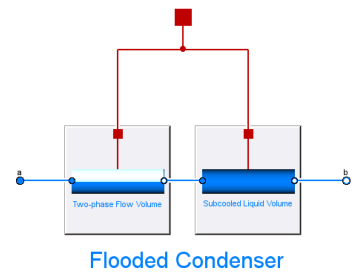

(d)

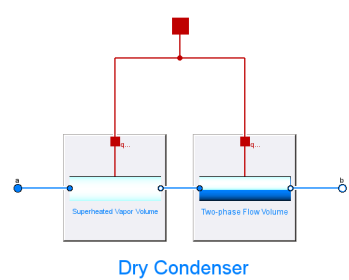

(f)
Figure 9: Condenser components

\subsection{Pipe Wall Component}

The pipe wall component includes the pipe wall model previously introduced in Section 2.6 adapted to support switching. The pipe wall component together with the Volume class depend on the geometry. The geometry is a partial class. Different geometries can be implemented by inheriting from the geometry class; the library already includes a cylindrical geometry model. Moreover, the pipe wall component inherits from a partial wall class, so different wall models can be implemented and used.

\subsection{The initialization problem}

The initialization problem is always a cumbersome task and it is especially difficult when considering inactive $\mathrm{CVs}$ in the initialization. For that reason, the initialization has been taken into account in the design of the MBMs library; the initialization options can be establish through the GUI in the initialization tab of evaporators and condensers. Fig. 10 shows the initialization options for a switching general evaporator. Here, it can be specified, which CVs are inactive in the initialization, the initial inlet pressure can be set as well as the initial outlet temperature (this value is 


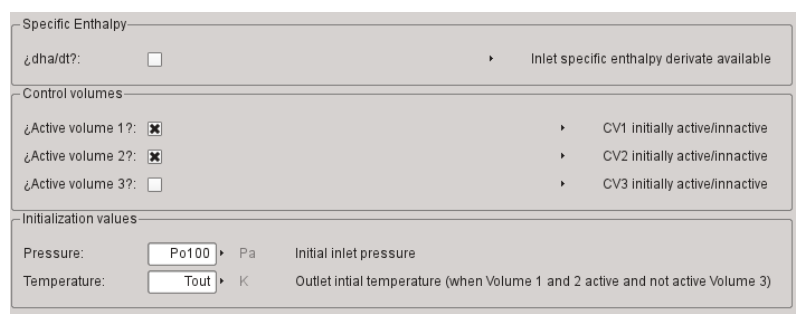

Figure 10: Initialization options for general evaporators

only required when the outlet fluid is two-phase flow), and it can be specified whether the inlet specific enthalpy time derivative is available. Sometimes when considering experimental data as input, this thermodynamic property may not be available and cannot be estimated, in which case the inlet specific enthalpy time derivative is set to zero.

\section{Simulation}

This section shows the simulation of the mathematical models previously introduced and implemented in the MBMs library. The medium in these simulations is the two-phase flow water-steam mixture from the Modelica Media library [20]. Dymola 2013 [6] has been the Modelica tool used for these simulations. The numerical solver used has been DASSL [23] and the relative tolerance has been set to $10^{-6}$. All of the developed models have been thoroughly tested in integrity and stability tests, however due to space limitation only a few can be presented in this article. A simulation test for a switching flooded evaporator was presented in [2].

\subsection{Model Integrity}

The simulation results must be verified and the governing equations of the model must be validated both in steady-state and in transient predictions. To this end, the mathematical model and library implementation results were compared to those of an independently developed finite volume model and code from the Modelica Fluid library [10] that belongs to the Modelica Standard Library 3.2. The Modelica Fluid library has been meticulously designed and tested and is widely used in the Modelica community.

Fig. 11 shows the outlet temperature for a test case considering a switching moving boundary general evaporator model from the MBMs library (dashed

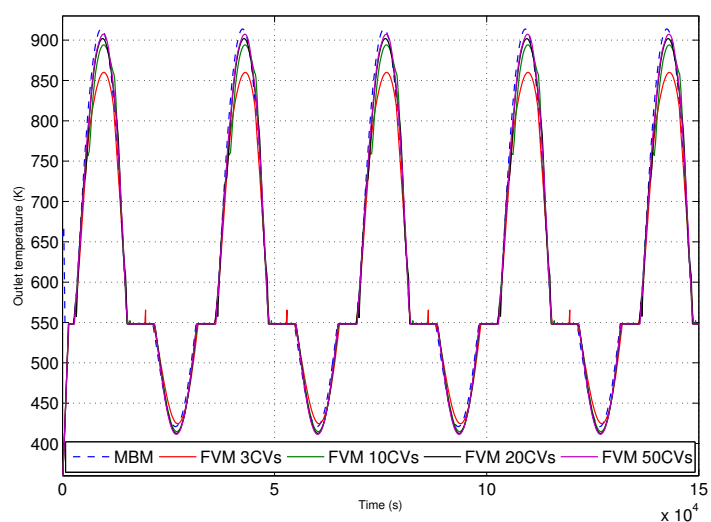

Figure 11: Integrity and stability test

\begin{tabular}{|l|c|c|}
\hline Model & CPU-Time (s) & State events \\
\hline MBM & 0.87 & 104 \\
FVM 3 CVs & 2.34 & 45 \\
FVM 10 CVs & 6.93 & 124 \\
FVM 20 CVs & 21.4 & 228 \\
FVM 50 CVs & 103 & 551 \\
\hline
\end{tabular}

Table 3: Simulation statistics

blue line) and finite volume models from the Modelica Fluid library considering different numbers of CVs $(3,10,20,50)$. It can be seen that the simulation results obtained with the MBMs library are in good agreement with those from the Modelica Fluid library and that the MBMs library model runs considerably faster (cf. Table 3), because the finite volume model requires at least $20 \mathrm{CV}$ s to yield acceptably accurate results.

\subsection{Model Stability}

Model stability, especially the switching stability, was checked by holding certain inputs constant during the simulation while varying sinusoidally others to force repeated switching. Variations in the heat flow rate, mass flow rate, inlet specific enthalpy and outlet pressure have been tested. Fig. 11 shows the outlet temperature in a switching general evaporator when varying sinusoidally the inlet heat flow rate over the pipe (cf. Fig. 12). The outlet fluid phase changes from subcooled liquid to two-phase flow (constant temperature) to superheated vapor. Fig. 13 shows the CV lengths in the moving boundary model where it can be observed, which CVs are inactive during the simulation (CVs with zero length), the length of the evaporator is $500 \mathrm{~m}$. 


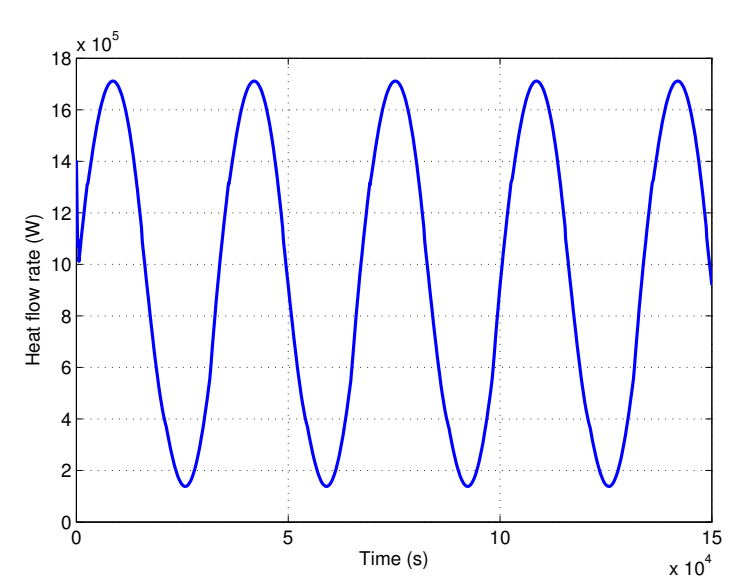

Figure 12: Heat flow rate over the pipe

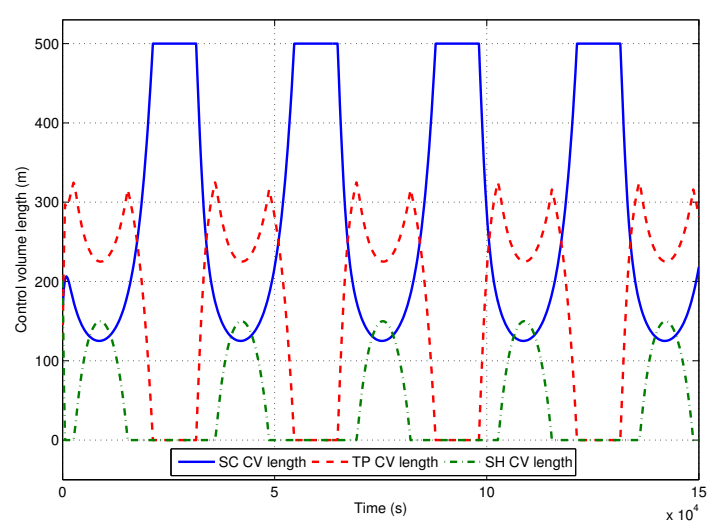

Figure 13: Control volume lengths

\section{Conclusions}

This paper details mathematical moving boundary models for heat exchangers, considering basic CVs and compound models: general, flooded and dry evaporators and condensers, independent of the two-phase flow medium. The pipe wall model is also shown. It is independent of the geometry, particularized for a cylindrical geometry in this paper. The switching criteria was also introduced allowing the disappearance of the CVs at the end of the heat exchanger. A new equation-based object-oriented Modelica library, the MBMs library, implementing all of the detailed models has been presented. This library provides models of different HTCs and FFMs. It also tackles the initialization problem, which is specially tough in the case of moving boundary models. The mathematical models and the MBMs library have been tested thoroughly using integrity and stability tests.

\section{Future work}

The MBMs library is currently still in its beta version, and some of the following open tasks will be considered for future library extensions: pressure drop in each $\mathrm{CV}$ and disappearance of $\mathrm{CVs}$ at the beginning of the heat exchanger. It is planned to use and validate the switching condenser models in the modeling of a double effect absorption heat pump in the ambit of the POWER project. The switching evaporator models are intended to be also validated in the HIBIOSOLEO project for the development of a direct steam generation linear Fresnel solar thermal power plant.

\section{References}

[1] S. Bendapudi, J. Braun, and E. Groll. A comparison of moving-boundary and finitevolume formulations for transients in centrifugal chillers. International Journal of Refrigeration, 31(8):1437-1452, December 2008.

[2] J. Bonilla, L.J. Yebra, S. Dormido, and F.E. Cellier. Object-Oriented Modeling of Switching Moving Boundary Models for Two-phase Flow Evaporators. In Proceedings MATHMOD 2012 - $7^{\text {th }}$ Vienna International Conference on Mathematical Modelling, 2012.

[3] J.C. Chen. Correlation for Boiling Heat Transfer to Saturated Fluids in Convective Flow. Industrial Engineering Chemistry Process Design and Development, 5(3):322-329, 1966.

[4] N.H. Chen. An Explicit Equation for Friction Factor in Pipe. Industrial \& Engineering Chemistry Fundamentals, 18(3):296-297, August 1979.

[5] C.F. Colebrook. Turbulent Flow in Pipes, with particular reference to the Transition Region between the Smooth and Rough Pipe Laws. Journal of the Institution of Civil engineers, 11(4):133-156, 1939.

[6] Dassault Systèmes. Dymola 2013 - Dynamic Modeling Laboratory. http://www.3ds.com/ products/catia/portfolio/dymola, 2012.

[7] M.M. Denn. Process Fluid Mechanics. Number 6. Prentice-Hall, Englewood Cliffs, 1980.

[8] M. Dhar and W. Soedel. Transient Analysis of a Vapor Compression Refrigeration System. In 
Proceedings of the $15^{\text {th }}$ International Congress of Refrigeration, pages 1031 - 1067, Venice, Italy, 1979.

[9] F.W. Dittus and L.M.K. Boelter. Heat transfer in automobile radiators of the tubular type. University of California Publications in Engineering, 2(1):443-461, 1930.

[10] R. Franke, F. Casella, M. Sielemann, K. Proelss, M. Otter, and M. Wetter. Standardization of Thermo-Fluid Modeling in Modelica.Fluid. In Proc. of the $7^{\text {th }}$ Int. Modelica Conference, pages 122-131, Italy, September 2009.

[11] V. Gnielinski. New equations for heat and mass transfer in turbulent pipe flow and channel flow. International Chemical Engineering, 2(16):359368, 1976.

[12] O. Goebel. Thermohydraulics of Direct Steam Generation. In Proceedings of the $9^{\text {th }}$ International Symposium on Solar Thermal Concentrating Technologies, Odeillo-Font-Romeu, 1998.

[13] M. Gräber, N.C. Strupp, and W. Tegethoff. Moving Boundary Heat Echanger Model and Validation Procedure. In Proceeding of EUROSIM, 2010 .

[14] E. Gungor and R.H.S. Winterton. A general correlation for flow boiling in tubes and annuli. International Journal of Heat and Mass Transfer, 29(3):351-358, 1986.

[15] S.E. Haaland. Simple and Explicit Formulas for the Friction Factor in Turbulent Pipe Flow. Journal of Fluids Engineering, 105(1):89-90, 1983.

[16] J.M. Jensen. Dynamic modeling of Thermo-Fluid Systems-With focus on evaporators for refrigeration. PhD thesis, Technical University of Denmark, 2003.

[17] J.M. Jensen and H. Tummescheit. Moving boundary models for dynamic simulations of two-phase flows. In Proc. of the $2^{\text {nd }}$ Int. Modelica Conference, 2002.

[18] R.W. Johnson. The Handbook of Fluid Dynamics. CRC Press, 1998.

[19] S.G. Kandlikar. A general correlation for saturated two-phase flow boiling heat transfer inside horizontal and vertical tubes. Journal of heat transfer, 112:219 - 228, 1990.
[20] Modelica Association. Modelica Standard Library 3.2, 2010.

[21] S.V. Patankar. Numerical Heat Transfer and Fluid Flow. Hemisphere, Washington,D.C, 1980.

[22] B.S. Petukhov. Heat Transfer and Friction in Turbulent Pipe Flow with Variable Physical Properties. Advances in Heat Transfer, 6(C):504-564, 1970.

[23] L.R. Petzold. A description of DASSL: a Diferential/Algebraic System Solver. Scientific Computing, pages 65-68, 1983.

[24] H. Schlichting and K. Gersten. Boundary-layer theory. Springer, 2000.

[25] M.M. Shah. Chart correlation for saturated boiling heat transfer: equations and further study. ASHRAE TransUnited States, 88(CONF820112-):185-196, 1982.

[26] E. Zarza. The Direct Steam Generation with Parabolic Collectors. The DISS project (in Spanish). PhD thesis, Escuela Superior de Ingenieros Industriales de Sevilla, Seville, Spain, November 2000

[27] W. Zhang and C. Zhang. A generalized moving-boundary model for transient simulation of dry-expansion evaporators under larger disturbances. International Journal of Refrigeration, 29(7):1119-1127, November 2006.

[28] D. Zimmer. Equation-Based Modeling of Variable-Structure Systems. PhD thesis, Swiss Federal Institute of Technology (ETH), 2010.

\section{Acknowledgments}

This work has been financed by CIEMAT research centre, by the INNPACTO project, Hibridación de tecnologías renovables en una planta de generación de energía. (HIBIOSOLEO), IPT-440000-2010-004 and the National Plan Project, Predictive COntrol techniques for efficient management of reneWable Energy micro-gRids. (POWER), DPI2010-21589-C05-02 of the Spanish Ministry of Economy and Competitiveness and FEDER funds. 